\title{
Four-Dimensional Computed Tomography: Clinical Impact for Patients with Primary Hyperparathyroidism
}

\author{
Nancy Perrier, MD \\ Department of Surgical Oncology, Unit 1484, University of Texas MD Anderson Cancer Center, Houston, TX
}

Tein et al. reviewed the experience at the Royal Melbourne Hospital regarding the utilization of fourdimensional computed tomography (4DCT) for primary hyperparathyroidism (PHPT). As the authors note, the dataset is not large enough to confirm a statistical change in the rate of directed parathyroidectomy [minimally invasive (MIP)] pre- and post-initiation of the modality, but does reveal that the technique is superb at gland localization. The data confirm that 4DCT provides an excellent suggestion as to where to start the operation in complex cases, and allowed 1:3 patients who would have otherwise had a bilateral neck exploration (BNE) to instead have a direct operation. Localization (usually defined as targeting the exact quadrant of the neck) decreases the extent of dissection, offers selective placement of incision, and avoids potential devascularization of the contralateral glands. Because the modality was only selectively used in difficult cases without concordance with other imaging techniques, it cannot be directly compared with sestamibi or ultrasound. The findings warrant consideration of $\mathrm{CT}$ as the primary imaging modality, with the goal of converting the study's $28 \%$ rate of BNE closer to the expected rate of $10-15 \%$ incidence of multigland disease.

The real clinical impact of 4DCT is on large-scale value pertaining to quality and cost. The cost of less time in the operating room, cost of less extensive dissection, and cost of shorter hospital stay should all be considered in calculating the value of the procedure. For these reasons, we recommend 4DCT as a primary imaging modality. Realizing that imaging location does not solely correlate with cure, the excellent rate of sustained eucalcemia in this population suggests that good imaging is a factor in valuebased care. In contrast to the proponents, it is recognized that there is a need for facilities to be experienced in understanding the timing of the injection protocol and the extra radiation exposure to the patient. Since its original description, one, rather than two, delayed washout images are recommended as this has no apparent bearing on image sensitivity. 4DCT does add cost but is actually less expensive than nuclear imaging studies.

The authors realize that this study is limited by not defining the extent of operation based on lateralization versus localization, non-segregation of primary versus reoperative cases, and no allowance for radiologist reading learning curve after implementation of this new technique. However, the paper mentions the observation of patient preference for $\mathrm{CT}$, and the data highlight the exceptional rate of $33 \%$ of patients avoiding a bilateral neck operation despite other imaging being discordant.

(C) Society of Surgical Oncology 2017

First Received: 15 September 2017;

Published Online: 9 November 2017

N. Perrier, MD

e-mail: nperrier@mdanderson.org 